\title{
A new internally heated diamond anvil cell system for time resolved optical and x-ray measurements
}

\section{Yimin Mijiti $^{a}$, Marco Perrri ${ }^{a}$, Jean Coquet ${ }^{b}$, Lucie Nataf ${ }^{b}$, Marco Minicucci $^{a}$, Angela Trapananti $^{a}$, Tetsuo Irifune $^{c}$, Francois Baudelet ${ }^{\mathrm{b}}$ and Andrea Di Cicco ${ }^{\mathrm{a}}$}

\author{
a Physics Division, School of Science and Technology, University of Camerino, Via Madonna delle Carceri 9, Camerino (MC), 62032, \\ Italy
}

${ }^{b}$ Synchrotron SOLEIL, L'Orme des Merisiers, Saint-Aubin, BP 48, 91192, Gif-sur-Yvettte Cedex, France

${ }^{c}$ Geodynamic Research Center, Ehime University, Matsuyama 790-8577, Japan

emin.mijiti@unicam.it

We have developed and tested a new internally heated diamond anvil cell (DAC) as reported in a recent paper published in Review of Scientific Instruments [1]. The system includes a portable vacuum chamber and was designed for routine performance of $\mathrm{x}$-ray and optical experiments. We have adopted a self-heating W/Re gasket design allowing for both sample confinement and heating. This solution proved to be very efficient to improve heating and cooling rates in a temperature regime up to $1500 \mathrm{~K}$. The system has been widely tested and calibrated under high-temperature conditions. The temperature distribution was measured by in situ optical measurements and resulted to be uniform within the typical uncertainty of the measurements $(5 \%$ at $1000 \mathrm{~K})$. XAS (x-ray absorption spectroscopy) of pure Ge at $3.5 \mathrm{GPa}$ were easily obtained in the $300 \mathrm{~K}-1300 \mathrm{~K}$ range, studying the melting transition and nucleation to the crystal phase. An original XAS-based dynamical temperature calibration procedure was developed and used to monitor the sample and diamond temperatures, indicating that heating and cooling rates in the $100 \mathrm{~K} / \mathrm{s}$ range can be easily achieved using this device.
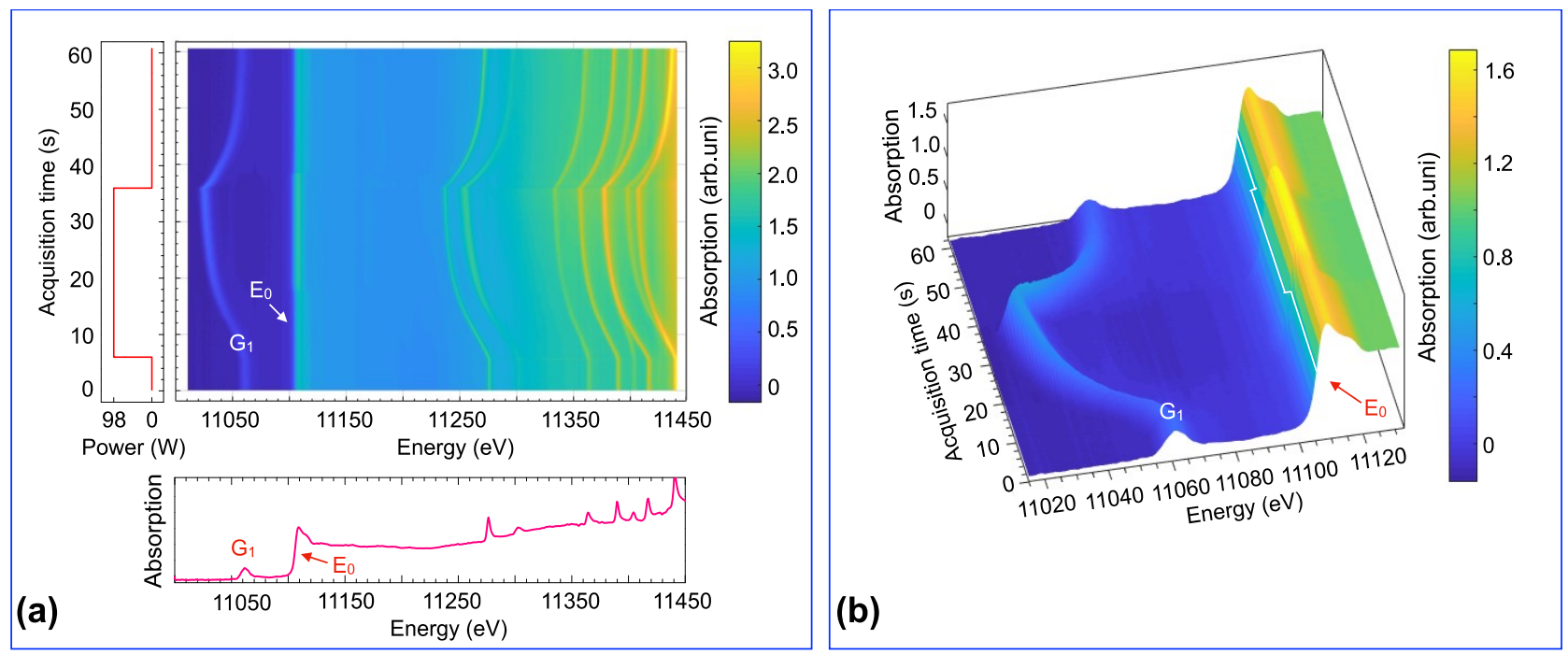

Figure 1. (a): 2D plot of the time resolved Ge $K$-edge XAS data presenting the absorption as function of photon energy and acquisition time (t). For clarity, the supplied electric power as function of time (square wave) is given at the left side of the figure. The Ge $K$-edge XAS spectrum at room temperature $(\mathrm{t}=0 \mathrm{~s})$ is shown at the bottom. (b): 3D visualization of time dependent evolution of the near-edge XAS and the first diamond glitch $\left(\mathrm{G}_{1}\right)$. The whole set of data was measured under nearly constant pressure at $\mathrm{P}=3.5 \mathrm{GPa}$.

[1] Y. Mijiti, M. Perri, J. Coquet, L. Nataf, M. Minicucci, T. Irifune, A. Trapananti, F. Baudelet, A. Di Cicco, Rev. Sci. Instrum. 91 , 085114 (2020)

Keywords: diamond Anvil cell, time resolution, high temperature, heating/cooling rates 